\title{
Fourier-Mukai Partners of Canonical Covers of Bielliptic and Enriques Surfaces
}

\author{
PaWel Sosna (*)
}

ABSTRACT - We prove that the canonical cover of an Enriques surface does not admit non-trivial Fourier-Mukai partners. We also show that the canonical cover of a bielliptic surface has at most one non-isomorphic Fourier-Mukai partner. The first result is then applied to birational Hilbert schemes of points and the second to birational generalised Kummer varieties.

Mathematics SubJect Classification (2010). 18E30, 11G10, 14J28

KEYworDs. Derived categories, Fourier-Mukai partners, bielliptic surfaces, Enriques surfaces

\section{Introduction}

In [11] Mukai discovered that an abelian variety $A$ and its dual abelian variety $\widehat{A}$ are always derived equivalent (or Fourier-Mukai partners), even though in general they are not even birational. Since this observation a lot of effort has been put into the investigation of possible Fourier-Mukai partners of a given variety $X$. It turns out that, for example, the derived category determines the variety if the canonical (or anti-canonical) bundle is ample. Thus, it is natural to consider the case where the canonical bundle is trivial or torsion. Since the derived category of an elliptic curve determines the curve, the case of surfaces is the next interesting one. If the Kodaira dimension of the surface is 0 , then, as is well known, there are four possibilities. The surface is either abelian, K3, Enriques or bielliptic. Bridgeland and Maciocia proved in [3] that an Enriques or a bielliptic

(*) Indirizzo dell'A.: Department Mathematik, Universität Hamburg, Bundesstrasse 55, 20146 Hamburg, Germany.

E-mail: pawel.sosna@math.uni-hamburg.de 
surface does not admit non-isomorphic Fourier-Mukai partners. In the other two cases quite a lot is known, see, for example, [6], [18] and [5]. Since the derived category often reflects geometry, it is interesting to ask what the relationship between the number of FM-partners of a variety and its canonical cover is. In this note we work in dimension 2 and prove the following result (see Propositions 3.1 and 4.3).

THEOREm 1.1. If $X$ is a Ko surface which covers an Enriques surface, then any surface derived equivalent to $X$ is isomorphic to $X$. If $A$ is an abelian surface which is the canonical cover of a bielliptic surface, then the only non-trivial Fourier-Mukai partner A can have is $\widehat{A}$.

Thus the additional geometric information imposes restrictions on the number of Fourier-Mukai partners. We will use the above result to establish that birational Hilbert schemes of points on K3 surfaces as above are automatically isomorphic, see Corollary 3.2; we also apply it to (one class of) Enriques manifolds in Corollary 3.3. Corollary 4.5 establishes a similar result in the case of birational generalised Kummer varieties.

The note is organised as follows: In Section 2 we recall the necessary facts about lattices and canonical covers and describe the known formulae for the number of Fourier-Mukai partners of a K3 resp. an abelian surface. In Section 3 we prove the first part of the theorem and Section 4 establishes the second part.

Throughout we work over the complex numbers and functors between derived categories are assumed to be derived.

\section{Preliminaries}

A lattice is a free abelian group $L$ of finite rank endowed with a symmetric non-degenerate Z-valued bilinear form $b$. A lattice is even if for any $l \in L$ the integer $b(l, l)$ is even. An isometry between two lattices is a group homomorphism preserving the bilinear forms. The group of self-isometries of a lattice $L$ will be denoted by $O(L)$. The dual $L^{*}$ is the $\operatorname{group} \operatorname{Hom}(L, Z)$ endowed with the natural extension of the bilinear form on $L$. There is an embedding $L \hookrightarrow L^{*}$ given by $l \mapsto b(-, l)$ and $L$ is called unimodular if the map is an isomorphism. An example of a unimodular lattice is the hyperbolic plane $U$, which is the group $Z^{2}$ endowed with the bilinear form which on the basis $e$ and $f$ is given by $e^{2}=f^{2}=0$ and $e f=1$. Another example is 
the unique positive definite even unimodular lattice $E_{8}$, see [1, Ch. I.2]. If $L$ is a lattice and $k \in Z$, then $L(k)$ denotes the same abelian group with the bilinear form multiplied by $k$. Given a sublattice $M$ of a lattice $L$, its orthogonal complement $M^{\perp}$ is the group of elements $l \in L$ satisfying $b(m, l)=0$ for all $m \in M$. We call a sublattice $M$ of a lattice $L$ primitive if $L / M$ is torsion-free.

The discriminant group of the lattice $L$ is by definition $A_{L}=L^{*} / L$. This is a finite group of order $|\operatorname{det}(L)|$ and $b$ induces a symmetric bilinear form $b_{L}: A_{L} \times A_{L} \rightarrow \mathrm{Q} / Z$ and a corresponding quadratic form $q_{L}: A_{L} \rightarrow$ Q/2Z. A lattice $L$ is $p$-elementary if $A_{L} \simeq(Z / p Z)^{a}$ for some natural number $a$.

Given a lattice $L$, its genus is the set $\mathcal{G}(L)$ of isometry classes of lattices $L^{\prime}$ such that $\left(A_{L}, q_{L}\right) \simeq\left(A_{L^{\prime}}, q_{L^{\prime}}\right)$ and the signature of $L^{\prime}$ is equal to the signature of $L$.

The following result of Nikulin, see [14, Thm. 14.4.2], will be used quite frequently in the sequel.

Proposition 2.1 (Nikulin). Let $T$ be an even indefinite nondegenerate lattice satisfying the following conditions:

(a) $\operatorname{rk}(T) \geq \operatorname{rk}\left(A_{T_{p}}\right)+2$ for all prime numbers $p \neq 2$.

(b) if $\operatorname{rk}(T)=l\left(A_{T_{2}}\right)$, then $q_{T_{2}}$ contains a component $u(2)$ or $v(2)$.

Then the homomorphism $O(T) \rightarrow O\left(A_{T}\right)$ is surjective and the genus of $T$ contains only one class. Here $A_{T_{p}}$ denotes the p-component of the finite abelian group $A_{T}, l$ denotes the number of generators, $u(2)$ is the discriminant group of the lattice $U(2)$ and $v(2)$ is described in [14].

We will frequently need the formulae for the number of Fourier-Mukai partners of a K3 surface or an abelian surface established in [6] and [5] respectively. Let $X$ be a $\mathrm{K} 3$ or abelian surface with transcendental lattice $T_{X}$ (sometimes written as $T(X)$ ) and period $\mathrm{C} \omega_{X}$. We define the group

$$
G_{\text {Hodge }}:=O_{\text {Hodge }}\left(T_{X}, \mathrm{C} \omega_{X}\right)=\left\{g \in O\left(T_{X}\right) \mid g\left(\mathrm{C} \omega_{X}\right)=\mathrm{C} \omega_{X}\right\} .
$$

It is known that the genus of a lattice with fixed rank and discriminant is a finite set. On the other hand, given a marking $\varphi$ for $X$, i.e. a lattice isomorphism between $H^{2}(X, Z)$ and the K3 lattice $\Lambda=U^{\oplus 3} \oplus E_{8}(-1)^{\oplus 2}(U$ and $E_{8}(-1)$ were defined above), we can use it to define an embedding $G_{\text {Hodge }} \hookrightarrow O(T)$, where $T=\varphi\left(T_{X}\right)$. Using that the discriminant groups of a lattice and its orthogonal complement are isomorphic, we get an action of $G_{\text {Hodge }}$ on $O\left(A_{T^{\perp}}\right)$. 
Consider a K3 surface $X$ and set $\mathcal{G}(N S(X))=\mathcal{G}(L)=\left\{L_{1}, \ldots, L_{k}\right\}$. Then the number of Fourier-Mukai partners $F M(X)$ of $X$ is given by

$$
|F M(X)|=\sum_{i=1}^{k}\left|O\left(L_{i}\right) \backslash O\left(A_{L_{i}}\right) / G_{\text {Hodge }}\right| .
$$

If $A$ is an abelian surface we have a surjective morphism

$$
\xi: F M(A) \rightarrow \mathcal{P}^{e q}\left(T(A), U^{\oplus 3}\right), B \mapsto \iota_{B},
$$

so that $\xi^{-1}\left(l_{B}\right)=\{B, \widehat{B}\}$ and where the set on the right is the set of $G_{H o d g e}{ }^{-}$ equivalence classes ( $G_{\text {Hodge }}$ is defined as above) of primitive embeddings of $T(A)$ into $U^{\oplus 3}$. We furthermore have

$$
\left|\mathcal{P}^{e q}\left(T(A), U^{\oplus 3}\right)\right|=\sum_{i=1}^{k}\left|O\left(L_{i}\right) \backslash O\left(A_{L_{i}}\right) / G_{\text {Hodge }}\right|,
$$

where the $L_{i}$ are the lattices in the genus of $N S(A)$.

We also need to recall the notion of canonical cover. Namely, let $X$ be a smooth projective variety with torsion canonical bundle $\omega_{X}$ whose order is $n$. The canonical cover $\widetilde{X}$ is the unique (up to isomorphism) smooth projective variety with trivial canonical bundle with an étale map $\pi: \widetilde{X} \rightarrow X$ of degree $n$ such that $\pi_{*} \mathcal{O}_{\widetilde{X}}=\bigoplus_{i=0}^{n-1} \omega_{X}^{i}$. Furthermore, there is a free action of the cyclic group $G=Z / n Z$ on $\widetilde{X}$ such that the morphism $\pi$ is the quotient morphism.

\section{K3 surfaces covering Enriques surfaces}

Recall that an Enriques surface is a compact complex surface $S$ of Kodaira dimension 0 with $H^{1}\left(S, \mathcal{O}_{S}\right)=H^{2}\left(S, \mathcal{O}_{S}\right)=0$. Any Enriques surface is projective, its canonical bundle is torsion of order 2 and the canonical cover of an Enriques surface is a K3 surface. Conversely, a quotient of a K3 surface by a fixed-point free involution is an Enriques surface. Note that we have an isomorphism $\operatorname{Pic}(S) \simeq H^{2}(S, Z)$ obtained from the exponential sequence. Dividing out torsion we get the lattice $E_{8}(-1) \oplus U$. Pullback to the covering K3 surface gives the lattice $E:=E_{8}(-2) \oplus U(2)$, which is often referred to as the Enriques lattice. An Enriques surface is generic if the Picard group of the covering K3 surface is precisely $E$.

By general results in [12] (which are based on Proposition 2.1) we know that a K3 surface of Picard rank $\geq 12$ does not have any Fourier-Mukai 
partners. Thus, if we consider K3 surfaces covering Enriques surfaces we only have to consider Picard ranks 10 and 11.

Proposition 3.1. Let $S$ be an Enriques surface and let $X$ be the covering Ko surface. If $Y$ is a surface such that $\mathrm{D}^{\mathrm{b}}(X) \simeq \mathrm{D}^{\mathrm{b}}(Y)$ (this forces $Y$ to be a Ko surface as well), then $X \simeq Y$.

Proof. By the Derived Torelli theorem for K3 surfaces the existence of a derived equivalence between $X$ and $Y$ is equivalent to the existence of a Hodge isometry between the transcendental lattices $T(X) \simeq T(Y)$. Let us begin with generic Enriques surfaces, that is, the Picard rank of the covering $\mathrm{K} 3$ is 10 . It is a fact that the orthogonal lattice of $E$ in the K3 lattice $A$ is isometric to $E \oplus U$, see (2.9) in [13]. Now, [13, Thm. 1.4] gives that any isometry of $E \oplus U$ extends to an isometry of $\Lambda$. By the Torelli theorem for K3 surfaces we conclude that $X \simeq Y$.

Ohashi [16, Prop. 3.5] classified the Néron-Severi lattices of K3 surfaces of Picard rank 11 covering an Enriques surface. We have the following two series

$$
\begin{aligned}
& F_{N}:=U(2) \oplus E_{8}(-2) \oplus\langle-2 N\rangle, \quad N \geq 2, \\
& G_{M}:=U \oplus E_{8}(-2) \oplus\langle-4 M\rangle, \quad M \geq 1 .
\end{aligned}
$$

In the second case, the Néron-Severi group contains the hyperbolic plane and hence any isometry of the transcendental lattice extends to the K3 lattice by [14, Thm. 14.4.4]. Therefore, the K3 surfaces belonging to the second case do not have any non-trivial FM-partners. Consider the lattices $F_{N}$. It is clear that for any $p \neq 2$ the rank of the $p$-component of the discriminant group is at most 1 . Hence, condition (a) in Proposition 2.1 is satisfied. One also easily sees that condition (b) is satisfied as well. Equation 2.1 gives the result.

Corollary 3.2. Let $X$ and $Y$ be two Ko surfaces covering Enriques surfaces and assume that the Hilbert schemes of $m$ points $(m \in \mathbb{N}$ ) $\operatorname{Hilb}^{m}(X)$ and $\operatorname{Hilb}^{m}(Y)$ are birational. Then there exists an isomorphism $\operatorname{Hilb}^{m}(X) \simeq \operatorname{Hilb}^{m}(Y)$.

Proof. The assumption implies that $\mathrm{D}^{\mathrm{b}}(X) \simeq \mathrm{D}^{\mathrm{b}}(Y)$, see [19, Prop. 10]. Now apply the above proposition.

Of course, the same argument works, for example, for Hilbert schemes of points on K3 surfaces of Picard rank at least 12 . 
We can apply this result to Enriques manifolds which were introduced in [15]. An Enriques manifold is a connected complex manifold which is not simply connected and whose universal cover is a hyperkähler manifold. A particular example is obtained as follows. Let $S$ be an Enriques surface and let $X$ be the K3 surface covering it. For any odd $n \geq 1$ the induced action of the group $G=Z / 2 Z$ (corresponding to the involution on $X$ ) on $\operatorname{Hilb}^{n}(X)$ is free and the quotient is an Enriques manifold $R$ with $\pi_{1}(R)=Z / 2 Z$.

Corollary 3.3. Let $R=\operatorname{Hilb}^{m}(X) / G$ and $R^{\prime}=\operatorname{Hilb}^{m}\left(X^{\prime}\right) / G$ be birational Enriques manifolds, where $X$ and $X^{\prime}$ cover generic Enriques surfaces. Then there exists an isomorphism $R \simeq R^{\prime}$.

Proof. The universal covers are birational as well and the claim follows at once from the previous corollary and the observation that under our assumption the surface $X\left(\simeq X^{\prime}\right)$ admits only one fixed-point free involution, see [16, Prop. 2.2].

The situation changes if one also considers twisted FM-partners of a given $\mathrm{K} 3$ surface $X$. Recall that given a $\mathrm{K} 3$ surface $X^{\prime}$ and a class $\alpha$ in the Brauer group of $X^{\prime}$, one can consider the abelian category of $\alpha$-twisted sheaves on $X^{\prime}$ and its bounded derived category $\mathrm{D}^{\mathrm{b}}\left(X^{\prime}, \alpha\right)$. A twisted FMpartner of $X$ is a twisted $\mathrm{K} 3$ surface $\left(X^{\prime}, \alpha\right)$ such that there is an equivalence $\mathrm{D}^{\mathrm{b}}\left(X^{\prime}, \alpha\right) \simeq \mathrm{D}^{\mathrm{b}}(X)$ (see, for example, [7] for more information). We have an explicit formula in [9] which allows us to compute the number of twisted FM-partners $\mathrm{FM}^{d}(X)$ of $X$ for any given order $d$ of $\alpha$. If the Néron-Severi lattice is 2-elementary, as is the case for the Enriques lattice, then [9, Cor. 4.5] states that there are no twisted FM-partners for $d \neq 1,2$. Applying this to a $\mathrm{K} 3$ surface $X$ with $\operatorname{Pic}(X)=E$, we have

$$
\mathrm{FM}^{2}(X)=\left|O_{\text {Hodge }}(T(X)) \backslash I^{2}\left(A_{T(X)}\right)\right|,
$$

where $I^{2}\left(A_{T(X)}\right)$ is the set of isotropic elements in $A_{T(X)}$ of order 2 . We know that the group $O_{\text {Hodge }}(T(X))$ is cyclic and that its Euler function value divides 12 , the rank of $T(X)$, see [6, Prop. B.1]. Thus, $\left|O_{\text {Hodge }}(T(X))\right| \leq 42$. On the other hand, it is easily checked that $I^{2}\left(A_{T(X)}\right)$ has more than 42 elements. Thus, the cover $X$ of a generic Enriques surface has a twisted Fourier-Mukai partner. It is to be expected that the same holds for higher Picard numbers.

If one twists $X$ as well, then for any natural number $N$ there exist $N$ non-isomorphic algebraic K3 surfaces $X_{1}, \ldots, X_{N}$ of Picard rank 20, which can be assumed to be Kummer surfaces, and Brauer classes $\alpha_{1}, \ldots, \alpha_{N}$ on 
these surfaces such that the twisted derived categories $\mathrm{D}^{\mathrm{b}}\left(X_{i}, \alpha_{i}\right)$ are all derived equivalent, see [7, Prop. 8.1]. Since any Kummer surface covers an Enriques surface by [8], we see that allowing twisting creates arbitrarily many twisted FM-partners.

\section{Abelian surfaces covering bielliptic surfaces}

Recall that a bielliptic surface is a complex projective surface $S$ of Kodaira dimension 0 with $H^{1}\left(S, \mathcal{O}_{S}\right)=\mathrm{C}$ and $H^{2}\left(S, \mathcal{O}_{S}\right)=0$. It turns out that any such surface is a quotient of a product of two elliptic curves $E \times F$ by the action of a finite group $G$. Note that we allow $E$ and $F$ to be isomorphic. The group $G$ acts on $E$ by translations and on $F$ in such a manner that $F / G \simeq \mathbb{P}^{1}$, so, in particular, it does not act by translations only. The canonical bundle of $S$ is torsion of order $2,3,4$ or 6 . In fact, there are only the following possibilities for $G$ and some of them include restrictions on $F$ (see [1, Ch. V.5]). We will write $A$ for the canonical cover $\widetilde{S}$.

(1) The group $G$ is cyclic of order $n=2,3,4$ or 6 . The order of $G$ is equal to the order of the canonical bundle of $S$ and the canonical cover $A$ is isomorphic to $E \times F$, where $F$ has complex multiplication for $n=3,4$ and 6 .

(2) The group $G$ is $Z / 3 Z \times Z / 3 Z$. The curve $F$ has complex multiplication, the order of the canonical bundle is 3 , so $A \simeq E \times F /(Z / 3 Z)$.

(3) The group $G$ is $Z / 2 Z \times Z / 2 Z$. Then $A=E \times F /(Z / 2 Z)$.

(4) The group $G$ is $Z / 4 Z \times Z / 2 Z$. The curve $F$ has complex multiplication and $A=E \times F /(Z / 2 Z)$.

Note that in the cases (2) and (4) the Picard rank of $\widetilde{S}$ is either 2 or 4 , depending on whether $E$ is isogenous to $F$ or not. Also recall that if an abelian surface has Picard rank 4, then it is isomorphic to a product of elliptic curves, see [22, Thm. 4.1].

We start with two preliminary lemmas.

Lemma 4.1. Let L be the lattice $U(3)$. Then the morphism $O(L) \rightarrow O\left(A_{L}\right)$ is surjective.

Proof. It is easy to see that $O(L)$ is isomorphic to $Z / 2 Z \oplus Z / 2 Z$, the isometries being the identity id, -id, the map $l$ which interchanges the two generators of the hyperbolic plane $e$ and $f$ and the composition - id $\circ l$. The 
group $A_{L}$ is $Z / 3 Z \oplus Z / 3 Z$. The bilinear form $b_{L}$ can be described as follows: The elements of the form $(x, 0)$ resp. $(0, x)$ are isotropic, $b_{L}(x, x)=\frac{1}{3}$ for all $x$ and $b_{L}(x, y)=\frac{2}{3}$ for $x \neq y, x \neq 0, y \neq 0$. The surjectivity of the canonical map follows by an easy computation.

\section{LEMma 4.2. The lattice U(3) is the only one in its genus.}

Proof. One can use the classification of indefinite two-dimensional lattices as found for example in [4, Ch. 15] and check that the discriminant forms of the other three two-dimensional lattices of determinant -9 are not isometric to the discriminant form of $U(3)$.

Proposition 4.3. Let $S$ be a bielliptic surface.Then the canonical cover $A$ of $S$ has at most one non-trivial FM-partner, namely $\widehat{A}$.

Proof. Firstly, let $A$ be an abelian surface which is isomorphic to a product of elliptic curves. Then any Fourier-Mukai partner of $A$ is isomorphic to $A$. Indeed, let $B$ be a FM-partner of $A$. By Orlov's results in [17] there exists a Hodge isometry $T(A) \simeq T(B)$ and, since by assumption the Néron-Severi group of $A$ contains a hyperbolic plane, we can again use [14, Thm. 14.4.4] to conclude that this isometry extends to a Hodge isometry $H^{2}(A, Z) \simeq H^{2}(B, Z)$. By [21, Thm. 1] this shows that $A \simeq B$ (since $A$ is selfdual being a product of elliptic curves). In particular, the canonical cover of a bielliptic surface does not have any non-trivial FM-partners in case (1). This also holds in the cases (2), (3) and (4) if $\rho(\widetilde{S})=4$.

We will now deal with the cases (3) and (4) with Picard $\operatorname{rank} 2$, thus $A$ be an abelian surface such that $N S(A)$ is isometric to $U(2)$. Then $A$ has precisely one non-isomorphic Fourier-Mukai partner, namely $\widehat{A}$. This holds because the lattice $U(2)$ is even, indefinite and 2-elementary. Therefore, by Proposition 2.1 any Hodge isometry of its orthogonal complement $T(A)$ extends to $H^{2}(A, Z)$. Using [21, Thm. 1] this implies that any non-trivial FM-partner of $A$ is either $A$ or $\widehat{A}$.

The next step is to consider (3) in the case that $\rho(\widetilde{S})=3$. First note, that $N S(E \times E)=U \oplus\langle-2\rangle$ for a curve $E$ without complex multiplication, but we can also have $E \times F$ with $F$ isogenous to $E$ and in this case $N S(E \times F)=U \oplus\langle-2 N\rangle$ for some $N \geq 1$. Dividing out Z/2Z gives a lattice of the form $L=U(2) \oplus\langle-4 N\rangle$. Arguing as in the last part of the proof of Proposition 3.1, we conclude that in this case there is also at most one non-trivial FM-partner. Lastly, we now turn to case (2). We only have to consider the case where $E$ is not isogenous to $F$, so $\rho(\widetilde{S})=2$. The Néron- 
Severi group of $E \times F$ is generated by $E \times\{0\}$ and $\{0\} \times F$ with the two generators spanning the hyperbolic plane $U$. It is easy to see that $N S(E \times F /(Z / 3 Z))=U(3)$. Combining Lemmas 4.1 and 4.2 with the counting formula recalled in Section 2 concludes the proof of the proposition.

REMARK 4.4. Two abelian varieties $A$ and $B$ are derived equivalent if and only if there exists a symplectic isomorphism $A \times \widehat{A} \simeq B \times \widehat{B}$, see [18]. If $A=E \times F$ and $B=E^{\prime} \times F^{\prime}$ we hence have $E \times F \times E \times F \simeq$ $E^{\prime} \times F^{\prime} \times E^{\prime} \times F^{\prime}$. This is not sufficient to conclude that $A \simeq B$ : In [20] Shioda gives a counterexample to such a statement even in smaller dimensions. Namely, there exist elliptic curves $C, C^{\prime}$ and $C^{\prime \prime}$ such that $C \times C^{\prime} \simeq C \times C^{\prime \prime}$ but nevertheless $C^{\prime} \approx C^{\prime \prime}$.

The above result has the following implication.

Corollary 4.5. If $A$ is an abelian surface as in the proposition and the generalised Kummer variety $K_{m}(A)(m \geq 2)$ is birational to $K_{m}(B)$ for some abelian surface $B$, then $B$ is either isomorphic to $A$ or $\widehat{A}$.

Proof. This follows at once, since the assumption implies that $\mathrm{D}^{\mathrm{b}}(A) \simeq \mathrm{D}^{\mathrm{b}}(B)$ (see the proof of [23, Prop. 3.3]).

REMARK 4.6. Similar to the K3-case we expect the situation to become more complicated when we consider twisted surfaces.

REMARK 4.7. An interesting question is whether any FM-partner of an abelian surface admitting a principal polarisation also admits a principal polarisation. This would follow from a stronger statement, namely that a principally polarised abelian surface does not have any non-trivial FMpartners: This is not entirely implausible by the above results for surfaces which are products of elliptic curves and by results in [18] which state that the statement holds in the generic case, that is, when $\operatorname{End}(A)=Z$. Namely, for a (not necessarily principally polarised) generic abelian variety $A$ the number of FM-partners of $A$ is equal to $2^{s(A)}$, where $s(A)$ is the number of prime divisors of $\operatorname{det}(N S(A)) / 2$.

REMARK 4.8. As a further example how the close geometric connection between a bielliptic surface $S$ and its canonical cover is reflected on the level of derived categories one can show that, if $n=2$, no object $E$ in $\mathrm{D}^{\mathrm{b}}(S)$ is 
rigid, that is, $\operatorname{Hom}^{1}(E, E) \neq 0$ for all $E$, where $\operatorname{Hom}^{i}(E, E):=\operatorname{Hom}(E, E[i])$. In particular, spherical and exceptional objects do not exist on such a bielliptic surface (recall that an object $E$ in the derived category $\mathrm{D}^{\mathrm{b}}(X)$ of a smooth projective variety $X$ of dimension $d$ is spherical if $E \otimes \omega_{X} \simeq E$ and $\operatorname{Hom}^{i}(E, E)=\mathbb{C}$ for $i=0, d$ and 0 otherwise; an object $E$ is called exceptional if $\operatorname{Hom}(E, E)=\mathrm{C}$ and $\operatorname{Hom}^{i}(E, E)=0$ for all $\left.i \neq 0\right)$. Indeed, the statement is true for the covering abelian surface $A$ by [2, Lem. 15.1]. Denoting the quotient morphism $A \rightarrow S$ by $\pi$, the statement follows from

$$
\begin{aligned}
\operatorname{Hom}^{i}\left(\pi^{*} E, \pi^{*} E\right) & =\operatorname{Hom}^{i}\left(E, \pi_{*} \pi^{*} E\right)=\operatorname{Hom}^{i}\left(E, E \otimes \pi_{*} \mathcal{O}_{\widetilde{X}}\right)= \\
& =\bigoplus_{k=0}^{n-1} \operatorname{Hom}^{i}\left(E, E \otimes \omega_{X}^{k}\right) .
\end{aligned}
$$

Note that the situation is completely different for Enriques surfaces. Indeed, any line bundle $L$ on an Enriques surface $X$ is an exceptional object since $\operatorname{Hom}^{i}(L, L)=\operatorname{Ext}^{i}(L, L) \simeq \operatorname{Ext}^{i}\left(\mathcal{O}_{X}, \mathcal{O}_{X}\right) \simeq H^{i}\left(X, \mathcal{O}_{X}\right)$. Also note that there are no spherical objects on a generic Enriques surface (see [10, Prop. 3.17]), but they do exist on non-generic ones.

Acknowledgments. I thank Daniel Huybrechts for his comments on a preliminary version of this paper and Martí Lahoz, Emanuele Macrì and Sven Meinhardt for useful discussions. This work was partially financially supported by the SFB/TR 45 'Periods, Moduli Spaces and Arithmetic of Algebraic Varieties' of the DFG (German Research Foundation).

\section{REFERENCES}

[1] W. Barth - K. Hulek - C. Peters - A. van de Ven, Compact complex surfaces, Springer, Berlin (2004).

[2] T. Bridgeland, Stability conditions on Ko surfaces, Duke Math. J. 141 (2008), pp. 241-291.

[3] T. BRidgeland - A. Maciocia, Complex surfaces with equivalent derived categories, Math. Z. 236 (2001), pp. 677-697.

[4] J. H. Conway - N. J. A. SloAne, Sphere packings, lattices and groups, 3rd ed., Springer, New York, 1999.

[5] S. Hosono - B. H. LiAn - K. OGUISO - S.-T. YAU, Kummer structures on a K3 surface: An old question of T. Shioda, Duke Math. J. 120 (2003), pp. 635-647.

[6] S. Hosono - B. H. LiAn - K. Oguiso - S.-T. YAU, Fourier-Mukai numbers of a Ko surface, In: Algebraic structures and moduli spaces, pp. 177-192. Amer. Math. Soc., Providence, RI (2004).

[7] D. Huybrechts - P. Stellari, Equivalences of twisted Ko surfaces, Math. Ann. 332 (2005), pp. 901-936. 
[8] J. H. Keum, Every algebraic Kummer surface is the Ko-cover of an Enriques surface, Nagoya Math. J. 118 (1990), pp. 99-110.

[9] S. MA, Twisted Fourier-Mukai number of a Ko surface, Trans. Amer. Math. Soc. 362 (2010), pp. 537-552.

[10] E. Macrì - S. Mehrotra - P. Stellari, Inducing stability conditions, J. Alg. Geom. 18 (2009), pp. 605-649.

[11] S. MukaI, Duality between $D(X)$ and $D(\widehat{X})$ and its application to Picard sheaves, Nagoya Math. J. 81 (1981), pp. 153-175.

[12] S. MukaI, On the moduli space of bundles on Ko surfaces, I, in: Vector bundles on algebraic varieties (Bombay, 1984), pp. 341-413, Tata Inst. Fund. Res., Bombay (1987).

[13] Y. Namikawa, Periods of Enriques surfaces, Math. Ann. 270 (1985), pp. 201222.

[14] V. V. NikULIN, Integral symmetric bilinear forms and some of their applications, Math. USSR Izv. 14 (1980), pp. 103-167.

[15] K. Oguiso - S. SchröER, Enriques manifolds, J. Reine Angew. Math. (to appear).

[16] H. OHASHI, On the number of Enriques quotients of a Ko surface, Publ. Res. Inst. Math. Sci. 43 (2007), pp. 181-200.

[17] D. OrLov, Equivalences of derived categories and Ko surfaces, J. Math. Sci. 84 (1997), pp. 1361-1381.

[18] D. Orlov, Derived categories of coherent sheaves on abelian varieties and equivalences between them, Izv. Math. 66 (2002), pp. 569-594.

[19] D. Ploog, Equivariant autoequivalences for finite group actions, Adv. Math. 216 (2007), pp. 62-74.

[20] T. SHIODA, Some remarks on abelian varieties, J. Fac. Sci. Univ. Tokyo Sect. IA 24 (1977), pp. 11-21.

[21] T. Shioda, The period map of abelian surfaces, J. Fac. Sci. Univ. Tokyo Sect. IA 25 (1977), pp. 47-59.

[22] T. SHioda - N. Mitani, Singular abelian surfaces and binary quadratic forms, In: Classification of algebraic varieties and compact complex manifolds, Springer, Berlin (1974), pp. 259-287.

[23] P. Stellari, Derived categories and Kummer varieties, Math. Z. 256 (2007), pp. $425-441$.

Manoscritto pervenuto in redazione il 16 Maggio 2012. 
